\title{
Hölder-Banach Space Analysis of the Bethe-Salpeter Equation
}

\author{
J. V. GReENMAN \\ Department of Mathematics, University of Essex, Colchester, Essex
}

Received July 29, 1969

\begin{abstract}
We find a Hölder Banach space in which the Bethe-Salpeter equation is a compact integral equation as it stands. We study the properties of the solution in preparation for an analysis of linear field theory models of 3-body amplitudes. In particular we study the properties of the Regge poles of the solution and prove the existence and uniqueness of on mass shell scattering amplitudes.
\end{abstract}

\section{Introduction}

We wish to consider the properties of linear field theory models for three-body amplitudes taking as input solutions of the two-body BetheSalpeter (BS) equation [1]. Previous work [2-12] on the BS equation has shown that the integral operator of that equation can always be related to a compact operator. This is achieved usually by either rotation [3] in the complex energy plane or by the use of spectral representations $[8,10]$, the end product being the discussion of an $L^{P}$ (Lebesgue) Banach space and the elimination of the most objectionable if not all singularities of the kernel. This is essential for numerical solution but results in an unnecessarily complicated discussion of the mathematical structure of the equation. In this paper we prove that the BS operator is compact as it stands. We follow the idea of Faddeev [13] and look for a suitable Hölder Banach space for which the assertion is true. We find such a space by first examining the spectral representation introduced by Wick [3] and exploited by Pagnamenta and Taylor [10]. The BS equation written as an equation for the spectral functions (Eq. (1.14)) has many features in common with the non-relativistic Lippmann-Schwinger equation [14] after removal of certain singularities by multiplicative similarity transformations (relations (2.2) and (2.4)). A suitable space in which to look for solutions of Eq. (1.14) is a simple generalisation of that introduced by Faddeev. This Banach space is denoted by $\bar{B}_{\mu \theta}^{(2)}$ with norm specified in relation (2.6). Eq. (1.14) is a compact integral equation in this space. The original BS equation is compact in the space $\tilde{B}_{\mu \theta}^{(2)}$ with norm specified in relation (2.7) generated from $\bar{B}_{\mu \theta}^{(2)}$. Construction of $\tilde{B}_{\mu \theta}^{(2)}$ from $\bar{B}_{\mu \theta}^{(2)}$ has the advantage that functions in $\tilde{B}_{\mu \theta}^{(2)}$ have sufficient analyticity for us to Wick rotate the BS equation for values of total 
energy below the elastic threshold and hence to relate properties of the solution above threshold to the whole body of information $[3,4,15]$ obtained by analysis of the simpler Wick rotated equation below threshold. Further, the spectral form sufficiently unscrambles the singularities to enable us to give a simple proof of, for example, the unitarity relation (Eq. (2.25)).

The argument in Section II establishing the central theorem is specifically for a $\phi^{3}$-theory in the ladder approximation with equal masses in the propogators, and in partial wave projection. These restrictions are made solely for simplicity of exposition. The result still holds with unequal masses and with any potential consistent with the assumed analyticity properties and certain boundedness conditions. With the more general potential some modification of the argument has to be made and this is sketched in Section III in the context of discussing the method of proof for the full BS equation.

In Section II we establish the main features of the movement with total energy of those poles of the meromorphic resolvent of the BS integral operator, considered as an operator valued function of complex angular momentum, that contribute to the scattering amplitude on the mass shell. We deduce that there is always an on mass shell solution of the BS equation in the elastic scattering region. For higher values of total energy we need to consider 3-body equations and this we intend to do in a further paper.

Throughout the analysis we will use the following notation (i) $Q=\left(q_{0}, \boldsymbol{q}\right)$, (ii) $q=|\boldsymbol{q}|$, (iii) $E=\left(E\right.$, 0 ), (iv) $Q_{ \pm}=Q \pm \frac{1}{2} E$, (v) $Q \cdot P$ $=q_{0} p_{0}-\boldsymbol{q} \cdot \boldsymbol{p}$, (vi) $Q \cdot Q=Q^{2}$, (vii) $\delta_{ \pm}\left(x^{2}-a^{2}\right)=\theta( \pm x) \delta\left(x^{2}-a^{2}\right)$. To avoid cluttering the equations with irrelevant constant multiplicative factors we will take $\lambda$ to denote the coupling constant and any constant multiple of that coupling constant. Further, in the arguments to establish inequalities between functions in the Hölder spaces we will use the convention of Faddeev [13] by denoting bounding constants by $C$, the occurrence of $C$ in any two statements not necessarily implying the equality of those constants.

\section{The Formalism}

We consider the Bethe-Salpeter equation in a $\phi^{3}$-theory in the ladder approximation, namely the equation,

$$
T=T_{0}+i \lambda K T
$$

where

(i) $D_{1}=(Q-P)^{2}-M^{2}$,

(ii) $D_{2}=\left(P+\frac{1}{2} E\right)^{2}-m^{2}$,

(iii) $D_{3}=\left(P-\frac{1}{2} E\right)^{2}-m^{2}$,

(iv) $T=T\left((Q-R)^{2}, Q_{ \pm}^{2}, R_{ \pm}^{2}, E\right)$, 
(v) $T_{0}=T_{0}\left((Q-R)^{2}\right)=(2 \pi)^{4} \lambda\left[(Q-R)^{2}-M^{2}\right]^{-1}$ is the Born term,

(vi) $K$ is the integral operator over the 4-momentum $P$ with kernel $K(Q|E| P)=\left(D_{1} D_{2} D_{3}\right)^{-1}$. For simplicity we take $m$ not equal to $M$. If $m$ were equal to $M$ then there are confluences of singularities that render the analysis more difficult. Our results remain valid in this case although the Banach spaces involved have more complicated norms.

The partial wave projection of Eq. (1.1) yields,

where

$$
T_{\ell}=T_{0 \ell}+i \lambda K_{\ell} T_{\ell}
$$

(i) $\boldsymbol{Q}=\left(q_{0}, q\right), \boldsymbol{R}=\left(r_{0}, r\right)$,

(ii) $\cos \theta_{q r}=(q r)^{-1}(\boldsymbol{q} \cdot \boldsymbol{r})$,

(iii) $T_{\ell}(Q|E| \boldsymbol{R})=\int_{-1}^{+1} d\left(\cos \theta_{q r}\right) P_{\ell}\left(\cos \theta_{q r}\right) T\left((Q-R)^{2}, Q_{ \pm}^{2}, R_{ \pm}^{2}, E\right)$,

(iv) $z_{Q P}=(2 q p)^{-1}\left[q^{2}+p^{2}+M^{2}-\left(q_{0}-p_{0}\right)^{2}\right]$,

(v) $K_{\ell}$ is an integral operator over the variables $\boldsymbol{P}$ with kernel $K_{\ell}(\boldsymbol{Q}|E| \boldsymbol{P})=(2 q)^{-1} p Q_{\ell}\left(z_{Q P}\right)\left(D_{2} D_{3}\right)^{-1} \cdot Q_{\ell}$ is the Legendre function of the second kind. The Froissart-Gribov continuation off the positive integers into the complex angular momentum plane is achieved by continuation of the $Q_{\ell}$ function in the kernel of (1.2) [16].

To find an appropriate Banach space in which to look for solutions of Eqs. (1.1) and (1.2) we will follow Wick [3] and Pagnamenta and Taylor [10] in exploiting the analyticity properties of $T$. To understand the properties of the consequent spectral functions we first consider the Feynman integral $T_{1}$ for the box diagram of Fig. 1. We know that the Fourier transform of the function $T_{1}$ in the variable $q_{0}$ has the form,

$$
\tilde{T}_{1}\left(x_{0}\right)=\theta\left(x_{0}\right) \tilde{\sigma}_{+}^{(1)}\left(x_{0}\right)+\theta\left(-x_{0}\right) \tilde{\sigma}_{-}^{(1)}\left(x_{0}\right) .
$$

Clearly,

$$
\begin{aligned}
& \tilde{\sigma}_{ \pm}^{(1)}\left(x_{0}\right)=-(2 \pi)^{\frac{3}{2}} \int_{-\infty}^{+\infty} d Q_{0} \int d^{3} p \delta^{ \pm}\left(D_{1}\right) e^{-i Q_{0} x_{0}} \sum_{i=2}^{4} \\
& \cdot \int_{-\infty}^{+\infty} d p_{0} e^{-i p_{0} x_{0}} \delta^{ \pm}\left(D_{i}\right)\left(\prod_{i \neq j} D_{j}\right)^{-1}
\end{aligned}
$$

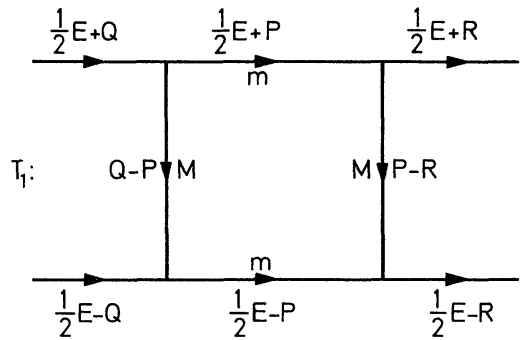

Fig. 1 
where $Q_{0}=q_{0}-p_{0}$ and $D_{4}=(P-R)^{2}-M^{2}$. The inverse Fourier transform of Eq. (1.4) yields,

$$
\sigma_{ \pm}^{(1)}\left(q_{0}\right)=-(2 \pi)^{2} \int d^{4} p \delta^{ \pm}\left(D_{1}\right) \sum_{i=2}^{4} \delta^{ \pm}\left(D_{i}\right)\left(\prod_{i \neq j} D_{j}\right)^{-1} .
$$

The supports of the three terms in the sum for $\sigma_{+}^{(1)}$ are given by,

$$
\begin{aligned}
& q_{0} \geqq-\frac{1}{2} E+\left(q^{2}+(m+M)^{2}\right)^{\frac{1}{2}}, \\
& q_{0} \geqq+\frac{1}{2} E+\left(q^{2}+(m+M)^{2}\right)^{\frac{1}{2}}, \\
& q_{0} \geqq r_{0}+\left((\boldsymbol{q}-\boldsymbol{r})^{2}+4 M^{2}\right)^{\frac{1}{2}} .
\end{aligned}
$$

The supports of the three terms in the sum for $\sigma_{-}^{(1)}$ are obtained from $(1.6 \mathrm{a}-\mathrm{c})$ by replacing $q_{0}$ and $r_{0}$ by $-q_{0}$ and $-r_{0}$. The relation between $T_{1}$ and $\sigma_{ \pm}^{(1)}$ is given by,

$$
T_{1}=i\left(B_{1}, B_{2}\right)\left(\begin{array}{l}
\sigma_{+}^{(1)} \\
\sigma_{-}^{(1)}
\end{array}\right)
$$

where $B_{1}$ is an integral operator over $q_{0}{ }^{\prime}$ with kernel $\left(q_{0}-q_{0}{ }^{\prime}+i \varepsilon\right)^{-1}$ and $B_{2}$ an integral operator over $q_{0}{ }^{\prime}$ with kernel $-\left(q_{0}-q_{0}{ }^{\prime}-i \varepsilon\right)^{-1}$.

We now wish to express the term $K T$ of Eq. (1.1) in the form (1.7) supposing that $T$ can be written in this form with the support (1.6). The Fourier transform of $K T$ for $x_{0}>0$ is given by

$$
\begin{aligned}
-i(2 \pi)^{\frac{1}{2}} \int d^{3} p \int_{-\infty}^{+\infty} d Q_{0} e^{-i Q_{0} x_{0}} \delta^{+}\left(D_{1}\right) & \\
& \cdot \int_{-\infty}^{+\infty} d p_{0} e^{-i p_{0} x_{0}}\left(D_{2} D_{3}\right)^{-1}\left(B_{1}, B_{2}\right)\left(\begin{array}{l}
\sigma_{+} \\
\sigma_{-}
\end{array}\right)
\end{aligned}
$$

if $T=i\left(B_{1}, B_{2}\right)\left(\begin{array}{l}\sigma_{+} \\ \sigma_{-}\end{array}\right)$. We calculate the integral over $p_{0}$ by completing the contour in the lower half plane (Fig. 2). We then take the inverse Fourier transform of Eq. (1.8) with the result that,

where

$$
K T=i\left(B_{1}, B_{2}\right)\left(\begin{array}{l}
(K T)_{+} \\
(K T)_{-}
\end{array}\right)
$$

$$
\begin{aligned}
(K T)_{+}= & -(2 \pi)^{2} i \int d^{4} p \delta^{+}\left(D_{1}\right) \\
& \cdot\left\{\left[D_{3}^{-1} \delta^{+}\left(D_{2}\right)+D_{2}^{-1} \delta^{+}\left(D_{3}\right)\right]\left(\bar{B}_{1}, B_{2}\right)\left(\begin{array}{l}
\sigma_{+} \\
\sigma_{-}
\end{array}\right)+\left(D_{2} D_{3}\right)^{-1} \sigma_{+}\right\}, \\
(K T)_{-}= & -(2 \pi)^{2} i \int d^{4} p \delta^{-}\left(D_{1}\right) \\
& \cdot\left\{\left[D_{3}^{-1} \delta^{-}\left(D_{2}\right)+D_{2}^{-1} \delta^{-}\left(D_{3}\right)\right]\left(B_{1}, \bar{B}_{2}\right)\left(\begin{array}{l}
\sigma_{+} \\
\sigma_{-}
\end{array}\right)+\left(D_{2} D_{3}\right)^{-1} \sigma_{-}\right\}
\end{aligned}
$$




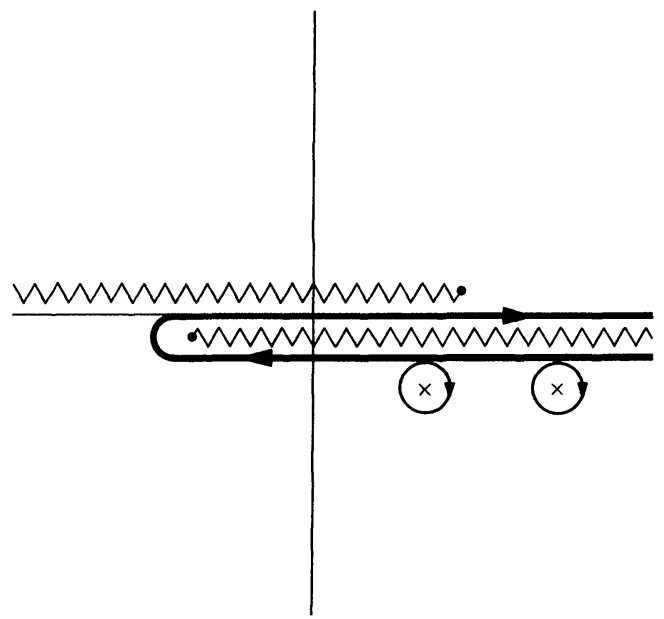

Fig. 2

where the bar above an operator indicates the complex conjugate operator. The spectral functions $\underset{\sim}{\sigma}=\left(\begin{array}{l}\sigma_{+} \\ \sigma_{-}\end{array}\right)$satisfy the equation,

$$
\sigma=\sigma^{0}+\lambda \tilde{K} \sigma
$$

where

(i) $V_{ \pm}$is an integral operator over 4-momentum with kernel $\delta^{ \pm}\left(D_{1}\right)$,

(ii) $D_{ \pm}$is an operator corresponding to multiplication by the function $\delta^{ \pm}\left(D_{2}\right) D_{3}^{-1}+\delta^{ \pm}\left(D_{3}\right) D_{2}^{-1}$

(iii) $D$ is an operator corresponding to multiplication by the function $\left(D_{2} D_{3}\right)^{-1}$,

(iv) $p_{0}{ }^{\min }\left(p_{0}{ }^{\text {max }}\right)$ is the minimum (maximum) value of $p_{0}$ for given $p, r, r_{0}$ of points lying in the support of $\sigma_{+}^{(1)}\left(\sigma_{-}^{(1)}\right)$,

(v) $\theta_{+}\left(\theta_{-}\right)$is the operator corresponding to multiplication by the step function $\theta\left(p_{0}-p_{0}{ }^{\min }\right)\left(\theta\left(p_{0}{ }^{\max }-p_{0}\right)\right)$,

(vi) $\tilde{V}=\left(\begin{array}{ll}V_{+}, & 0 \\ 0, & V_{-}\end{array}\right)$,

(vii) $\tilde{\theta}=\left(\begin{array}{ll}\theta_{+}, & 0 \\ 0, & \theta_{-}\end{array}\right)$,

(viii) $\tilde{W}=\left(\begin{array}{ll}D_{+} \bar{B}_{1}, & D_{+} B_{2} \\ D_{-} B_{1}, & D_{-} \bar{B}_{2}\end{array}\right)$,

(ix) $\tilde{D}=\left(\begin{array}{ll}D, & 0 \\ 0, & D\end{array}\right)$,

(x) $\tilde{K}_{1}=\tilde{V} \tilde{W} \tilde{\theta}$,

(xi) $\tilde{K}_{2}=\tilde{V} \tilde{D} \tilde{\theta}$,

(xii) $\tilde{K}=\tilde{K}_{1}+\tilde{K}_{2}$. 


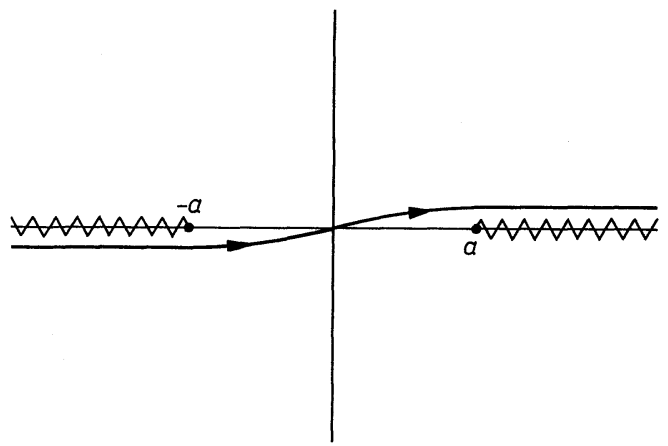

Fig. 3

To project Eq. (1.12) into partial waves and thence to continue into the complex angular momentum plane it is probably simplest to return to Eq. (1.2) with kernel $K_{\ell}$ already continued into the complex plane, and then to apply to $K_{\ell}$ the method of argument used in the progression from Eqs. (1.3) to (1.12). In the Fourier transform equivalent to (1.4) we have an integration of the form,

$$
\int_{-\infty}^{+\infty} d k_{0} e^{-i k_{0} x_{0}} Q_{\ell}\left(z_{P Q}\right)
$$

where $k_{0}=p_{0}-q_{0} . Q_{\ell}\left(z_{P Q}\right)$ has cuts in the ranges $-\infty<k_{0}<-a$, $a<k_{0}<\infty$ where $a=\left[(p-q)^{2}+M^{2}\right]^{\frac{1}{2}}$ with the contour of integration as given in Fig. 3. Hence for $x_{0}>0$ we pick up the right hand discontinuity and for $x_{0}<0$ the left hand discontinuity. The projected spectral functions $\sigma^{\ell}$ satisfy the equation

$$
\sigma^{\ell}=\sigma^{0 \ell}+\lambda \tilde{K}_{\ell} \sigma^{\ell}
$$

$\tilde{K}_{\ell}$ is obtained from $\tilde{K}$ by replacing the operator $\tilde{V}$ by $\tilde{V}_{\ell}=\left(\begin{array}{ll}V_{+}^{\ell}, & 0 \\ 0, & V_{-}^{\ell}\end{array}\right)$ where $V_{+}^{\ell}\left(V_{-}^{\ell}\right)$ is an integral operator whose kernel is the right (left) hand discontinuity of $Q_{\ell}\left(z_{P Q}\right)$.

\section{Compactness of the Integral Operator of the Partial Wave Bethe-Salpeter Equation}

The natural Banach space to choose to render Eq. (1.14) well defined is the Hölder space, which, for a one variable problem, is the complete space $B_{\mu \theta}^{(1)}$ of bounded continuous functions with norm,

$$
\|f\|_{\mu \theta}=\sup _{\substack{x, \delta \\|\delta|<1}}(1+|x|)^{\theta}\left\{|f(x)|+\frac{|f(x+\delta)-f(x)|}{|\delta|^{\mu}}\right\} .
$$


Besides the added complication of an extra variable, solutions of Eq. (1.14) have divergencies other than in the Born term. These can be removed by multiplicative similarity transformations. We define $S$ and $T$ to be the operators,

where

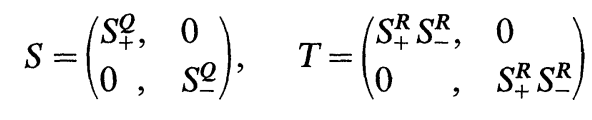

(i) $\phi=\left[\frac{1}{4} E^{2}-m^{2}\right]^{\frac{1}{2}}$,

(ii) $F_{ \pm}^{Q}=q_{0} \mp\left[(q-\phi)^{2}+M^{2}\right]^{\frac{1}{2}}$,

(iii) $G_{ \pm}^{Q}=q_{0} \mp\left[(q+\phi)^{2}+M^{2}\right]^{\frac{1}{2}}$,

(iv) $S_{ \pm}^{Q}$ denotes multiplication by the function,

$$
S_{ \pm}^{Q}=q^{\frac{1}{2}}\left(1+q^{\frac{1}{2}}\right)^{-1}\left|F_{ \pm}^{Q}\right|^{\frac{1}{2}}\left(1+\left|F_{ \pm}^{Q}\right|^{\frac{1}{2}}\right)^{-1}\left|G_{ \pm}^{Q}\right|^{\frac{1}{2}}\left(1+\left|G_{ \pm}^{Q}\right|^{\frac{1}{2}}\right)^{-1} .
$$

We define new spectral functions $\underline{\sim}^{\ell}=S T \sigma^{\ell}$ satisfying the equation,

$$
y^{\ell}=y^{0 \ell}+\lambda K_{\ell}^{\prime} y
$$

where $K_{\ell}^{\prime}=S \tilde{K}_{\ell} S^{-1}$. We introduce the complete Banach space $B_{\mu \theta}^{(2)}$ of functions $f(\boldsymbol{Q})$ with norm,

$$
\begin{aligned}
\|f\|_{\mu \theta}^{(2)}= & \sup _{\substack{q_{0}, q \\
|d|<1}}\left(1+\left|q_{0}\right|\right)^{\theta}(1+q)^{\theta} \\
& \cdot\left\{\left|f\left(q_{0}, q\right)\right|+\frac{\left|f\left(q_{0}+\delta q_{0}, q+\delta q\right)-f\left(q_{0}, q\right)\right|}{|d|^{\mu}}\right\}
\end{aligned}
$$

where $d=\left[\left(\delta q_{0}\right)^{2}+(\delta q)^{2}\right]^{\frac{1}{2}}$ and the complete Banach space $\bar{B}_{\mu \theta}^{(2)}$ of two component functions $f(Q)=\left(\begin{array}{l}f_{+}(Q) \\ f_{-}(Q)\end{array}\right)$ with norm

$$
\|f\|_{\mu \theta}^{(2)}=\left\|f_{+}\right\|_{\mu \theta}^{(2)}+\left\|f_{-}\right\|_{\mu \theta}^{(2)} .
$$

Further, we introduce the complete Banach space $\tilde{B}_{\mu \theta}^{(2)}$ of functions $f(Q)$ of the form $f=\alpha T_{0 \ell}+g$ where $\alpha$ is a constant and $g$ is analytic in the cut $q_{0}$ plane and of the form $g=\left(B_{1}, B_{2}\right) S^{-1} T^{-1}\left(\begin{array}{l}g_{+} \\ g_{-}\end{array}\right)$with norm

$$
\|f\|=|\alpha|+\|g\|_{\mu \theta}^{(2)} \text {. }
$$

In the space $\bar{B}_{\mu \theta}^{(2)}$ Eq. (2.4), on iteration, is a compact integral equation. In the space $\tilde{B}_{\mu \theta}^{(2)}$ Eq. (1.2) is a compact integral equation. The proof of these assertions is given in the Appendix and is based on the following lemma:

Lemma 1. A sequence of functions bounded in the norm $B_{\mu^{\prime} \theta^{\prime}}^{(2)}$ is compact in $B_{\mu \theta}^{(2)}$ where $\theta<\theta^{\prime}, \mu<\mu^{\prime}$. We have the immediate corollary that an operator which maps any bounded infinite sequence of functions in $B_{\mu \theta}^{(2)}$ into a bounded sequence in $B_{\mu^{\prime} \theta^{\prime}}^{(2)}$ where $\theta<\theta^{\prime}, \mu<\mu^{\prime}$ is compact. 
The proof that our operator behaves in this fashion is given in a series of Lemmas in the Appendix. We list these lemmas in a sufficiently imprecise form to render their essential nature obvious.

Lemma 2. If $f(x, y) \in B_{\mu \theta}^{(2)}$ then,

$$
g(y)=\int_{a}^{\infty} d x \frac{f(x, y)}{b-x \pm i \varepsilon} \quad a \gtrless b
$$

belongs to $B_{\mu^{\prime} \theta}^{(1)}$ where $\mu^{\prime}<\mu$ but can be taken arbitrarily close.

Lemma 3. If $f(x) \in B_{\mu \theta}^{(1)}$ then

belongs to the space $B_{1 \theta}^{(1)}$.

$$
g(y)=\int_{y-a}^{y+a} d x f(x)
$$

Lemma 4. If $f(x, y) \in B_{\mu \theta}^{(2)}$ then,

$$
g(x, y)=\iint_{A(x, y)} d x^{\prime} d y^{\prime} \frac{f\left(x,{ }^{\prime}, y^{\prime}\right)}{\left(x^{\prime}-a \pm i \varepsilon\right)\left(y^{\prime}-b \pm i \varepsilon\right)}
$$

belongs to $B_{10}^{(2)}$ provided,

(i) the function $h(x, y)$, where $h(x, y)=0$ is the equation of the boundary of $A(x, y)$, is a differentiable function of $x, y$.

(ii) this boundary never touches the lines $x=a, y=b$.

Lemma 5. $g^{(1) \ell} \in \bar{B}_{\mu \frac{1}{2}}^{(2)}, T_{1 \ell} \in \tilde{B}_{\mu \frac{1}{2}}^{(2)}$ where (i) $T_{1 \ell}$ is the partial wave projection of the Feynman integral corresponding to the box diagram of Fig. 1 (ii) $\sigma^{(1) \ell}$ are the spectral functions of $T_{1 \ell}$ (iii) $\mu$ is less than but can be chosen arbitrarily close to $\frac{1}{2}$.

We then have the following theorem:

Theorem. The integral operator $K_{\ell}^{\prime}\left(K_{\ell}\right)$ of Eq. (2.4) (Eq. (1.2)) is a compact operator in the space $\bar{B}_{\mu \theta}^{(2)}\left(\tilde{B}_{\mu \theta}^{(2)}\right)$ with $\mu<\frac{1}{2}, \frac{1}{4}<\theta<\frac{1}{2}$ if $\ell \in \mathscr{D}$ where $\mathscr{D}$ is the domain defined by the constraint $\operatorname{Re} \ell>-\frac{1}{2}$ and $\operatorname{Im} \ell$ is arbitrarily bounded. Further, the Eq. (2.4) (Eq. (1.2)) is a compact integral equation in $\bar{B}_{\mu \theta}^{(2)}\left(\tilde{B}_{\mu \theta}^{(2)}\right)$ if $\ell \in \mathscr{D}$.

Since $K_{\ell}$ is analytic in the domain $\mathscr{D}$ the resolvent operator $R_{\ell}=\left[I-i \lambda K_{\ell}\right]^{-1}$ is meromorphic in $\mathscr{D}$. Precisely,

Lemma 6. If $\ell \rightarrow K$ is an analytic operator valued function defined on $\mathscr{D}$ and compact for each $\ell \in \mathscr{D}$ and if $\mathscr{D}$ is connected then either $\left[I-i \lambda K_{\ell}\right]$ has a bounded inverse for no point in $\mathscr{D}$ or else this inverse exists except at a countable number of isolated points in $\mathscr{D}$.

The proof is given in Ref. [17], part I, page 592.

We can now rigorously derive the usual properties of Regge poles and by use of the Fredholm alternative prove the existence of a unique solution to the Eq. (1.14) for $E^{2}$ in the range $4 m^{2}<E^{2}<(2 m+M)^{2}$. In 
fact, suppose for $E^{2}$ in this range there exists an eigenfunction $g$ of $\tilde{K}_{\ell}$ in Eq. (1.14) for $\ell$ real, that is

$$
\sigma=\lambda \tilde{K}_{\ell} \sigma .
$$

Taking the imaginary part of this equation we find that,

$$
(\sigma-\bar{\sigma})=\lambda\left(\tilde{K}_{\ell}-\overline{\tilde{K}}_{\ell}\right) \bar{\sigma}+\lambda \tilde{K}_{\ell}(\sigma-\bar{\sigma}) .
$$

For there to be a solution $(\sigma-\bar{\sigma})$ to this equation with $\left(\tilde{K}_{\ell}-\overline{\tilde{K}}_{\ell}\right) \bar{\sigma}$ considered as the inhomogeneous term, the inhomogeneous term must be orthogonal to all those elements of the dual space which are the eigensolutions of the dual equation, in particular orthogonal to

$$
\begin{aligned}
& \left(\begin{array}{l}
B_{1} \\
B_{2}
\end{array}\right) D\left(B_{1}, B_{2}\right) \sigma ; \\
& \sigma^{T}\left(\begin{array}{l}
B_{1} \\
B_{2}
\end{array}\right) D\left(B_{1}, B_{2}\right) \tilde{V_{\ell}} \tilde{\Delta}\left(\begin{array}{ll}
B_{2}, & B_{2} \\
B_{1}, & B_{2}
\end{array}\right) \bar{g}=0
\end{aligned}
$$

where (i) $\tilde{\Delta}=\left(\begin{array}{ll}\delta & 0 \\ 0 & \delta\end{array}\right)$, (ii) $\delta$ denotes the operator corresponding to multiplication by the function $\delta^{+}\left(D_{2}\right) \delta^{-}\left(D_{3}\right)$. Since

$$
\sigma^{T}\left(\begin{array}{l}
B_{1} \\
B_{2}
\end{array}\right) D\left(B_{1}, B_{2}\right) \tilde{V}_{\ell}=\sigma^{T}\left(\begin{array}{l}
B_{1} \\
B_{2}
\end{array}\right)
$$

Eq. (2.21) reduces to,

$$
\int d \boldsymbol{P} \phi \delta \bar{\phi}=0
$$

where $\phi=p\left(B_{1}, B_{2}\right) \sigma$. Necessarily $\phi(\boldsymbol{P})=0$ if $\boldsymbol{P}=(0, \phi)$. Clearly, this does not eliminate the possibility of poles in $R_{\ell}$ on the real axis for $4 m^{2}<E^{2}<(2 m+M)^{2}$ but it does eliminate the possibility of poles in $R_{\ell}$ on the real axis that contribute to the scattering amplitude on the mass shell as we now argue. In the neighbourhood of a pole $\ell=\ell_{0}\left(E^{2}\right)$ in the resolvent $R_{\ell}$ the solution $T_{\ell}$ of Eq. (1.2) can be written in the form

$$
\sum \eta \frac{\phi(\boldsymbol{Q}) \phi(\boldsymbol{R})}{\ell-\ell_{0}\left(E^{2}\right)}+\tilde{T}_{\ell}(\boldsymbol{Q}|E| \boldsymbol{R})
$$

where $\tilde{T}_{\ell}$ is analytic in a neighbourhood of $\ell_{0}\left(E^{2}\right), \eta$ is a multiplicative constant, $\phi$ is an eigenfunction of Eq. (1.2) for $\ell=\ell_{0}\left(E^{2}\right)$ and the sum is over all such eigenfunctions. The residue of the pole of $T_{\ell}$ is zero when $T_{\ell}$ is put on the mass shell. The solution of Eq. (1.2) evaluated on the mass shell exists, therefore, for all values of $E^{2}$ in the range $\left(4 m^{2},(2 m+M)^{2}\right)$.

The method of separating Eq. (1.14) into real and imaginary parts can also be used to derive the unitarity relation [18]. Specifically

$$
\sigma^{\ell}-\bar{\sigma}^{\ell}=\lambda\left(\tilde{K}_{\ell}-\overline{\tilde{K}}_{\ell}\right) \sigma^{\ell}+\lambda \tilde{K}_{\ell}\left(\sigma^{\ell}-\bar{\sigma}^{\ell}\right) .
$$


Considering this equation as an equation for $\sigma^{\ell}-\bar{\sigma}^{\ell}$ with inhomogeneous term $\lambda\left(\tilde{K}_{\ell}-\tilde{K}_{\ell}\right) \bar{\sigma}^{\ell}$ we obtain the relation

$$
\sigma^{\ell}-\bar{\sigma}^{\ell}=\lambda R_{\ell}\left(\tilde{K}_{\ell}-\overline{\tilde{K}}_{\ell}\right) \bar{\sigma}^{\ell}
$$

Operating on the left with $i\left(B_{1}, B_{2}\right)$ we find,

$$
T_{\ell}(\boldsymbol{Q}|E| \boldsymbol{R})-\bar{T}_{\ell}(\boldsymbol{Q}|E| \boldsymbol{R})=\lambda \varrho(E) T_{\ell}(\boldsymbol{Q}|E| \boldsymbol{P}) \bar{T}_{\ell}(\boldsymbol{P}|E| \boldsymbol{R})
$$

where $\boldsymbol{P}=(0, \phi)$, the points $\boldsymbol{Q}, \boldsymbol{R}$ do not lie in the support of $g$ and $\varrho(E)$ is the two body phase space factor. Relation (2.22) follows immediately from this unitarity relation.

For $E^{2}>(2 m+M)^{2}$ we do not possess sufficient positivity to argue that those poles of $R_{\ell}$ that contribute to $T_{\ell}$ on the mass shell stay off the real axis. It may be that in models that contain more inelasticity than the ladder model this positivity will be regained. We can say, however, that as $E^{2}$ tends to infinity all poles move out of the domain $\mathscr{D}$ and that the poles never move to infinity in $\mathscr{D}$ whatever value $E^{2}$ takes.

\section{Concluding Remarks}

We can treat the full Bethe-Salpeter equation (1.1) in a similar fashion by looking for solutions within a Hölder Banach space of functions $\tilde{B}_{\mu \theta}^{(4)}$ defined over four dimensional momentum space. In the ladder approximation the methods outlined in Lemmas $2-5$ are not directly applicable since now the integrations are reducible to integrals over surfaces in a three dimensional space that move with the external parameters. One can get over this difficulty by iterating the operator once to obtain an operator whose potential corresponds to the box diagram of Fig. 1. The integrations are now over fixed regions of fixed manifolds of at least three-dimensions. The lemma needed to prove that Eq. (1.1) is a compact integral equation is a multi-dimensional generalization of the following basic lemma:

Lemma 7. If $f(y)$ belongs to $B_{\mu \theta}^{(1)} ; g(x, y)$ to $B_{\mu^{\prime} \theta^{\prime}}^{(2)}$ then

$$
I(x)=\int_{0}^{\infty} d y g(x, y) \frac{1}{(y-a \pm i \varepsilon)} f(y)
$$

belongs to $B_{\mu^{\prime}, \theta^{\prime}}^{(1)}$ where $\mu^{\prime \prime}$ is less than but can be chosen arbitrarily close to $\mu^{\prime}$.

Further, the analysis, as in the partial wave case, is extendable to complex values of $E^{2}$ by taking the cuts in the complex $q_{0}$ plane parallel to the real axis from the threshold branch points $\pm \frac{1}{2} E \mp\left[q^{2}+m^{2}\right]^{\frac{1}{2}}$. Eq. (1.1) is a compact equation and its resolvent $R(\lambda, E)$ is a meromorphic operator in $\lambda$ and meromorphic in the cut $E^{2}$ plane, and the solutions are continuous on to the real axis in $E^{2}$. For $E^{2}<4 m^{2}$ the poles in $R(\lambda, E)$ 
in $\lambda$ lie necessarily [15] on the positive real axis with an accumulation point at infinity. For $E^{2}$ in the interval $\left(4 m^{2},(2 m+M)^{2}\right)$ there are no poles on the real axis which contribute to the scattering amplitude on the mass shell.

Acknowledgement. I would like to thank Professor J. G. Taylor and Dr. N. T. Peck for their help in the course of this work.

\section{Appendix}

The method of proof of the lemmas leading to the main theorem of the paper is a generalisation of the arguments of Faddev [13]. It seems unnecessary here to give full proofs to all lemmas. We will give the proof of Lemma 2 from which it should be clear how the proofs to the other lemmas run.

For simplicity we suppose $\boldsymbol{R}$ is such that the support of $\underset{\sim}{\mathcal{~ o f ~ E q . ~ ( 2 . 4 ) ~}}$ lies in the regions

$$
q_{0} \geqq-\frac{1}{2} E+\left[q^{2}+(m+M)^{2}\right]^{\frac{1}{2}}, \quad q_{0} \leqq \frac{1}{2} E-\left[q^{2}+(m+M)^{2}\right]^{\frac{1}{2}} .
$$

Further we suppose $\ell \in \mathscr{D}$.

Lemma 2. If $v_{ \pm}$belong to $B_{\mu \theta}^{(2)}\left(\mu<\frac{1}{2}, \theta<\frac{1}{2}\right)$ and if $0<E<(M+2 m)$ then the functions $\left.\left(\frac{p}{1+p}\right) T\left(B_{1} \theta_{+}, B_{2} \theta_{-}\right)\left(\begin{array}{c}\sigma_{+} \\ \sigma_{-}\end{array}\right)\right|_{D_{i}=0}(i=2,3)$ belong to $B_{\mu^{\prime} \theta^{\prime}}^{(1)}$ where $\theta^{\prime}\left(\mu^{\prime}\right)$ is less than but may be chosen arbitrarily close to $\theta(\mu)$.

Proof. We must consider integrals of the form,

where

$$
I_{\left(\varepsilon_{1}, \varepsilon_{2}\right)}(v)=\left.\int_{a+(v)}^{\infty} d u^{\prime} \frac{\sigma_{+}^{T}\left(u^{\prime}, v\right)}{a-u^{\prime} \pm i \varepsilon}\right|_{a=b_{\left(\varepsilon_{1}, \varepsilon_{2}\right)}(v)}
$$

(i) $u=p_{0}-p, v=p$,

(ii) $\sigma_{+}^{T}=T \sigma_{+}$,

(iii) $a_{+}(v)=-\frac{1}{2} E-v+\left[v^{2}+(m+M)^{2}\right]^{\frac{1}{2}}$,

(iv) $b_{\left(\varepsilon_{1}, \varepsilon_{2}\right)}(v)=\varepsilon_{1} \cdot \frac{1}{2} E-v+\varepsilon_{2}\left[v^{2}+m^{2}\right]^{\frac{1}{2}}$.

$\varepsilon_{1}, \varepsilon_{2}$, take the values \pm 1 . The integrand of $I_{(++)}$(v) possesses a pole at $u^{\prime}=b_{(++)}$and square root singularities from $S^{-1}$. The integral of $I_{(-+)}(v)$, however, has only the square root singularities.

We first argue that $I_{(++)}(v)$ is bounded. We write

where,

$$
I_{(++)}(v)=I_{(++)}^{(1)}(v)+I_{(++)}^{(2)}(v)
$$

$$
\begin{aligned}
& I_{(++)}^{(1)}(v)=\left[\int_{a_{+}}^{b_{(++)}-1} d u^{\prime}+\int_{b_{(+)+1}}^{\infty} d u^{\prime}\right]\left[\frac{\sigma_{+}^{T}\left(u^{\prime}, v\right)}{b_{(++)}-u^{\prime} \pm i \varepsilon}\right], \\
& I_{(++)}^{(2)}(v)=\int_{b_{(++)}-1}^{b_{(++)}} d u^{\prime}\left[\frac{\sigma_{+}^{T}\left(u^{\prime}, v\right)}{b_{(++)}-u^{\prime} \pm i \varepsilon}\right] .
\end{aligned}
$$


Since $\left|v_{+}\left(u^{\prime}, v\right)\right|<C(1+v)^{-\theta}\left(1+\left|u^{\prime}\right|\right)^{-\theta}, I_{(++)}^{(1)}(v)$ is clearly bounded and majorised by $C(1+v)^{-\theta}$. We write $I_{(++)}^{(2)}(v)$ in the form,

$$
\begin{aligned}
I_{(++)}^{(2)}(v)= & \int_{b_{(++)-1}}^{b_{(++)}^{+1}} d u^{\prime}\left[\frac{\sigma_{+}^{T}\left(b_{(++)}, v\right)}{b_{(++)}-u^{\prime} \pm i \varepsilon}\right] \\
& +\int_{b_{(++)-1}}^{b_{(++)}+1} d u^{\prime}\left[\frac{\sigma_{+}^{T}\left(u^{\prime}, v\right)-\sigma_{+}^{T}\left(b_{(++)}, v\right)}{b_{(++)}-u^{\prime} \pm i \varepsilon}\right]
\end{aligned}
$$

The first term is explicitly integrable, and majorized by $C(1+v)^{-\theta}$. The modulus of the second term is no greater than,

$$
C \int_{b_{(++)}-1}^{b(++)+1} d u^{\prime}\left|b_{(++)}-u^{\prime}\right|^{\mu-1}(1+v)^{-\theta}
$$

since

$$
\left|v_{+}\left(u^{\prime}, v\right)-v_{+}\left(b_{(++)}, v\right)\right|<C\left|b_{(++)}-u^{\prime}\right|^{\mu}\left(1+u^{\prime}\right)^{-\theta}(1+v)^{-\theta} .
$$

The result,

follows.

$$
\left|I_{(++)}(v)\right| \leqq C(1+v)^{-\theta}
$$

We now consider the differentiability of the function $I_{(++)}(v)$.

$$
\begin{aligned}
I_{(++)}^{(2)}(v+\delta)-I_{(++)}^{(2)}(v)= & \int_{b_{(++)}(v+\delta)-1}^{b_{(++)}(v+\delta)+1} d u^{\prime} \frac{\sigma_{+}^{T}\left(u^{\prime}, v+\delta\right)}{\left[b_{(++)}(v+\delta)-u^{\prime} \pm i \varepsilon\right]} \\
& -\int_{b_{(++)}(v)+1}^{b_{(++)}(v)-1} d u^{\prime} \frac{\sigma_{+}^{T}\left(u^{\prime}, v\right)}{\left[b_{(++)}(v)-u^{\prime} \pm i \varepsilon\right]} \\
= & \int_{-1}^{+1} \frac{d y}{(y \mp i \varepsilon)}\left[\sigma_{+}^{T}\left(y+b_{(++)}(v), v\right)\right. \\
& \left.-\sigma_{+}^{T}\left(y+b_{(++)}(v+\delta), v+\delta\right)\right] \\
= & \int_{-1}^{+1} \frac{d y}{(y \mp i \varepsilon)}\left[\sigma_{+}^{T}\left(b_{(++)}(v), v\right)\right. \\
& \left.-\sigma_{+}^{T}\left(b_{(++)}(v+\delta), v+\delta\right)\right] \\
& +\int_{-1}^{+1} \frac{d y}{(y \mp i \varepsilon)}\left[\sigma_{+}^{T}\left(y+b_{(++)}(v), v\right)\right. \\
& -\sigma_{+}^{T}\left(b_{(++)}(v), v\right)-\sigma_{+}^{T}\left(y+b_{(++)}(v+\delta), v+\delta\right) \\
& \left.+\sigma_{+}^{T}\left(b_{(++)}(v+\delta), v+\delta\right)\right] .
\end{aligned}
$$

The first term is clearly majorised by $C(1+v)^{-\theta}|\delta|^{\mu}$. To majorise the second term we need the result,

$$
\begin{aligned}
\left|v_{+}\left(u^{\prime}, v^{\prime}\right)-v_{+}\left(u^{\prime}, v\right)-v_{+}\left(u, v^{\prime}\right)+v_{+}(u, v)\right| \\
\leqq C(1+|u|)^{-\theta}(1+v)^{-\theta}\left|u^{\prime}-u\right|^{\mu(1-\lambda)} \mid v^{\prime}-v^{\mu \lambda}
\end{aligned}
$$


where $0 \leqq \lambda \leqq 1$. This lemma is proved in Appendix 1 of Ref. [13]. Hence the second term is majorised by $C|\delta|^{\mu^{\prime}}(1+v)^{-\theta}$ where $\mu^{\prime}$ is less than $\mu$ but may be taken arbitrarily close to $\mu$. Similar arguments lead to the bounds,

and

$$
\left|I_{(++)}^{(1)}(v+\delta)-I_{(++)}^{(1)}(v)\right| \leqq C|\delta|^{\mu}(1+v)^{-\theta}
$$

$$
\left|I_{(++)}(v+\delta)-I_{(++)}(v)\right| \leqq C|\delta|^{\mu^{\prime}}(1+v)^{-\theta}
$$

where $\mu^{\prime}$ is less than $\mu$ but may be taken arbitrarily close to $\mu$. We have identical bounds on $I_{(--)}(v)$. For $I_{(-+)}(v)$, however, $a_{+}(v)-b_{(-+)}(v)$ tends to zero as $v$ tends to infinity necessitating the slightly weaker bounds,

$$
\begin{aligned}
\left|I_{(-+)}(v)\right| & \leqq C(1+v)^{-\theta^{\prime}}, \\
\left|I_{(-+)}(v+\delta)-I_{(-+)}(v)\right| & \leqq C|\delta|^{\mu^{\prime}}(1+v)^{-\theta^{\prime}},
\end{aligned}
$$

where $\theta^{\prime}\left(\mu^{\prime}\right)$ is less than but can be taken arbitrarily close to $\theta(\mu)$. Identical bounds hold for $I_{(+-)}(v)$.

If $E>M+2 m$ the curve $u=b_{(-+)}(v)$ intersects the support of $\sigma_{-}$and $I_{(-+)}(v)$ belongs to $B_{\mu^{\prime} \theta^{\prime}}^{(1)}$ only after the removal of a logarithmic factor

$$
\ln \left\{v-\frac{1}{2 E}\left[E^{2}-M^{2}\right]^{\frac{1}{2}}\left[E^{2}-(M+2 m)^{2}\right]^{\frac{1}{2}}\right\} \text {. }
$$

\section{References}

1. Bethe, H., Salpeter, E. E.: Phys. Rev. 84, 1232 (1951);

Gell-Mann, M., Low, F. E.: Phys. Rev. 84, 350 (1951);

Schwinger, J.: Proc. natl. Acad. Sci. (Wash.) 37, 455 (1951).

2. Nakanishi, N.: J. Math. Phys. 4, 1229 (1963).

3. Wick, G. C.: Phys. Rev. 96, 1124 (1954).

4. Cutkosky, R.: Phys. Rev. 96, 1135 (1954).

5. Kemmer, N., Salam, A.: Proc. roy. Soc. (London) A 230, 266 (1955).

6. Graves-Morris, P. R.: Phys. Rev. Letters 16, 201 (1966).

7. Levine, M., Tjon, J., Wright, J.: Phys. Rev. Letters 16, 962 (1966).

8. Kramer, G., Meetz, K.: Commun. math. Phys. 3, 29 (1966).

9. Taylor, J. G.: Nuovo Cimento, Suppl. 3, 857 (1964).

10. Pagnamenta, A., Taylor, J. G.: Phys. Rev. Letters 17, 218 (1966).

11. Broido, M. M., Taylor, J. G.: J. Math. Phys. 10, 184 (1969).

12. Greenman, J. V.: Nuovo Cimento 58 B, 421 (1968).

13. Faddeev, L. D.: Mathematical aspects of the three-body problem in the quantum scattering theory. Israel Program for Scientific Translations, Jerusalem 1965.

14. Lippmann, B. A., Schwinger, J.: Phys. Rev. 79, 469 (1950).

15. Ciafaloni, M., Menotti, P.: Phys. Rev. 140, B 929 (1965).

16. Lee, B. W., Sawyer, R. F.: Phys. Rev. 127, 2266 (1962).

17. Dunford, N., Schwarz, J. T.: Linear operators. New York: Interscience 1958.

18. Alessandrini, V. A., Omnes, R. L.: Phys. Rev. 139, B 167 (1965).

J. V. Greenman

Department of Mathematics

University of Essex

Colchester, Essex, Great Britain 